\title{
2- Dominator Coloring for Various Graphs in Graph Theory
}

\author{
A. Sangeetha Devi ${ }^{1}$, M. M. Shanmugapriya ${ }^{2}$ \\ ${ }^{1}$ Research Scholar, Department of Mathematics, Karpagam University, Coimbatore-21, India \\ ${ }^{2}$ Assistant Professor, Department of Mathematics, Karpagam University, Coimbatore-21, India
}

\begin{abstract}
Given a graph $G$, the dominator coloring problem seeks a proper coloring of $G$ with the additional property that every vertex in the graph dominates at least 2-color class. In this paper, as an extension of Dominator coloring such that various graph using 2dominator coloring has been discussed.
\end{abstract}

Keywords: 2-Dominator Coloring, Barbell Graph, Star Graph, Banana Tree, Wheel Graph

\section{Introduction}

In graph theory, coloring and dominating are two important areas which have been extensively studied. The fundamental parameter in the theory of graph coloring is the chromatic number $\chi(\mathrm{G})$ of a graph $\mathrm{G}$ which is defined to be the minimum number of colors required to color the vertices of $\mathrm{G}$ in such a way that no two adjacent vertices receive the same color. If $\chi(G)=k$, we say that $G$ is k-chromatic.

A dominating set $\mathrm{S}$ is a subset of the vertices in a graph such that every vertex in the graph either belongs to $S$ or has a neighbor in $\mathrm{S}$. The domination number is the order of a minimum dominating set. Given a graph $\mathrm{G}$ and an integer k, finding a dominating set of order $\mathrm{k}$ is NP-complete on arbitrary graphs [2], [4].

Graph coloring is used as a model for a vast number of practical problems involving allocation of scarce resources (e.g., scheduling problems), and has played a key role in the development of graph theory and, more generally, discrete mathematics and combinatorial optimization. A graph has a dominator coloring if it has a proper coloring in which each vertex of the graph dominates every vertex of some color class. The dominator chromatic number $\chi_{\mathrm{d}}(\mathrm{G})$ is the minimum number of color classes in a dominator coloring of a graph $\mathrm{G}$. A $\chi_{\mathrm{d}}(\mathrm{G})$ - coloring of $\mathrm{G}$ is any dominator coloring with $\chi_{\mathrm{d}}(\mathrm{G})$ colors. Our study of this was motivated by [9] and [7].

\section{Various Kinds of Graphs in Graph Theory}

\section{Theorem 2.1}

Let $S_{1, n}$ be a star graph, C $\left[S_{1, n}\right]$ beacentralgraphofstargraph, then $\chi_{d, 2}\left\{\mathrm{C}\left[S_{1, n}\right]\right\}$ $\leq n+2, n \geq 3$.

Proof:

Let $U_{0}$ be the root vertex of $\mathrm{G}$. Let $\left\{v_{0}, v_{1}, v_{2}, \ldots . v_{n-1}\right\}$ be the remaining vertices of $G$. By definition of central graph $C(G)$, subdivide the each edge exactly once and joining all the non-adjacentvertices of G. Let $w_{0}, w_{1}, w_{2}, \ldots \ldots w_{n-1}$ be the subdividing vertices of $G$. By definition of 2-dominator coloring, assign color $C_{0}$ to the root vertices $u_{0}$. Now color of all the pendent vertices of $G$ by,$n^{\text {ee }}$ different colors. Introduce two new colors $, C_{i},{ }_{,}, C_{j}$ "ealternatively to the subdividing vertices of $G, C_{0}$ will dominates ${ }_{,} C_{i},{ }_{,}, C_{j}{ }^{e c}$. Also all the pendent verticies of $G$ dominates at least two color class. i.e, $1+n+2 \leq n+3$. Therefore $\chi_{d, 2}\left\{\mathrm{C}\left[S_{1, n}\right]\right\} \leq n+$ $3, n \geq 3$.

\section{Theorem 2.2}

Let $S_{1, n}$ be a star graph, then $\chi_{d, 2}\left(S_{1, n}\right)=2, n \geq 2$.

Proof:

Let $S_{1, n}$ be a star graph by 2 dominator coloring and from theorem 3.2 and 3.7[9], the assignment of colors to the vertices of $G$, May dominates itself and it forms a color class. Thus $\chi_{d, 2}\left(S_{1, n}\right)=2$.

\section{Theorem 2.3}

Let $G=B\left(K_{n}, K_{n}\right)$ where $n=3,4,5, \ldots$ Then $\chi_{d, 2}[B$ $\left.\left(K_{n}, K_{n}\right)\right]=n$.

\section{Proof:}

Let $G=B\left(K_{n}, K_{n}\right)$ be a Barbell graph. Now divide the vertices of $G$ be $U$ and $V$. Let $U=\left\{u_{0}, u_{1}, u_{2}, \ldots, u_{n-1}\right\}$ and $V=\left\{v_{0}, v_{1}, v_{2}, \ldots . v_{n-1}\right\}$ respectively. By definition of Barbell graph, $G$ is obtained by connecting two complete graphs $K_{n}$ by $a_{n}$ bridge $e_{i}$. Using theorem 1.2 [2] and 3.1 [3],2-dominator coloring in any complete graph all the vertices must contain at least $\mathrm{n}$ colors. Let the color vertex $u_{0}$ by $c_{0}, u_{1}$ by $c_{1}, u_{2}$ by $c_{2}, \ldots, u_{n}$ by $c_{n-1}$. Similarly, color of the vertex $v_{0}$ by $c_{0}, v_{1}$ by $c_{1}, v_{2}$ by $c_{2}, \ldots, v_{n}$ by $c_{n-1}$. Thus a bridge , $e_{i}{ }^{\text {ec }}$ between $U$ and $V$ will receive the color ,,$c_{0} c_{n-1}$. ${ }^{e}$. Hence no two adjacent vertices have same color. Also every vertex of $G$ dominates at least two color classes.

i.e. $\chi_{d, 2}\left[B\left(K_{n}, K_{n}\right)\right]=n, n=3,4,5, \ldots$.

\section{Theorem 2.4}

Let $\mathrm{G}=P_{2} \square P_{n}, n \geq 2$, then $\cdot \chi_{d, 2}(G)=4$.

\section{Proof:}

Let $\mathrm{G}=P_{2} \quad \square$ be a ladder graph, $n \geq 2$ (i.e. two rails or paths connected by rungs). Now color the vertices in path $P_{1}$ by two different colors $c_{0}, c_{1}$ alternatively. Similarly color. The vertices in path $P_{2}$ by another new two different colors

\section{Volume 5 Issue 4, April 2016}




\section{International Journal of Science and Research (IJSR) \\ ISSN (Online): 2319-7064}

Index Copernicus Value (2013): 6.14 | Impact Factor (2015): 6.391

$c_{2}, c_{3}$ alternatively. Therefore the every rung $P_{n}$ between the paths $P_{1}$ and $P_{2}$ will receive the color $c_{0}, c_{2}$ and $c_{1}, c_{3}$ alternatively. Hence by definition of 2 dominator coloring $\chi_{d, 2}\left(P_{2} P_{n}\right)=4, \mathrm{n} \geq 2$.

\section{Theorem 2.5}

Let $G=B_{m, n}, m, n \geq 2$ be a banana tree then $\chi_{d, 2}\left(B_{m, n}\right)=2$

\section{Proof:}

Let $B_{m, n}$ be a banana tree of order $m, n \geq 2$ ie $(n, m)$ - banana tree is a graph created by connecting a leaf of each of $m$ copies of n-star graph with a pendent vertex and it is different from all stars. Thus from theorem 3.2 and 3.7[9], $\chi_{d, 2}\left(B_{m, n}\right)=2$.

\section{Theorem 2.6}

If $\mathrm{G}=F_{n, k}, \mathrm{n}, \mathrm{k} \geq 2$, then $\chi_{d, 2}(G)=2$

\section{Proof:}

Let $\mathrm{G}=F_{n, k}, \mathrm{n}, \mathrm{k} \geq 2$, be a fire cracker graph i.e $F_{n, k}$ is a graph connecting $\mathrm{n}, \mathrm{k}-$ states by an leaf. By definition of 2 dominator coloring and by use of theorem 3.2 and 3.7[9], every vertex may dominates its own class. Thus $\chi_{d, 2}\left[F_{n, k}\right]=$ 2.

\section{Theorem 2.7}

If $\mathrm{G}=D_{n}^{(m)}, n$ be the number of vertex $m$ be the copies of complete graph, $n, m \geq 3$, then, $\chi_{d, 2}\left[D_{n}^{(m)}\right]=n$.

\section{Proof}

Let $\mathrm{G}=D_{n}^{(m)}$ be a wind mill graph and it is obtained of connecting $\mathrm{m}$ copies of $K_{n}$ with the common vertex. By the definition of 2- dominator coloring from theorem 1.2 and $3.1[3] D_{n}^{(m)}$ needs at least $\mathrm{n}$ colors and no two adjacent vertices will receive same color. Thus $\chi_{d, 2}\left[D_{n}^{(m)}\right]=n$, $\mathrm{n} \geq 3,4,5, \ldots$

\section{Theorem 2.8}

If $G=G_{n}$ be any gear graph then $\chi_{d, 2}(G)=4, n=3,4,5,7, \ldots$

\section{Proof:}

Let $G=G_{n}$ be a gear graph or bipartite wheel graph.

Let $\mathrm{u}_{1}$ be the root vertex of $G$.

Let $v_{1}, v_{2}, \ldots . v_{n}$ be the main vertex of $G$.

Let $w_{1}, w_{2}, \ldots \ldots w_{n}$ be the sub vertices of $G$.

Procedure for 2- dominator coloring of $G$ :

Case 1: For $n=3, \chi_{d, 2}\left(G_{3}\right)=4$.

Let a root vertex $\mathrm{u}$ is assigned by the color $c_{0}$. Now assign color to vertex $v_{0}$ by $c_{0}, v_{1}$ by $c_{1}, v_{2}$ by $c_{2}$ Similarly $w_{0}$ by $c_{0}, w_{1}$ by $c_{1}, w_{2}$ by $c_{2}$ respectively. Therefore every vertex must dominate at least two color class. Hence $\chi_{d, 2}(G)=4$.

Case 2: For $n=4,5,6, \ldots, \chi_{d, 2}\left(G_{3}\right)=4$.

Assign color $\mathrm{C}_{0}$ to the vertex $\mathrm{u}_{0}$. Now color $\mathrm{C}_{1}, \mathrm{C}_{2}$ alternatively to the vertices $v_{0}, v_{1}, \ldots \ldots, v_{n-1}$. Hence a root vertex $u_{0}$ colored by $C_{0}$ will dominates the color classes $C_{1}$ and $C_{2}$ respectively. The vertices $w_{0}, w_{1}, \ldots \ldots, w_{n-1}$ is assigned by the color $C_{3}$ such that $C_{3}$ will dominates the color classes
$\mathrm{C}_{1}$ and $\mathrm{C}_{2}$ in $G$. By definition of 2-Dominator coloring the vertices of $G$ must dominates at least two color classes. Hence $\chi_{d, 2}\left(G_{3}\right)=4, n=4,5,6, \ldots \ldots$

Theorem 2.9

If $G=W_{n}$ be a wheel graph then

$\chi_{d, 2}\left(W_{n}\right)=\left\{\begin{array}{l}3, n=6,8,10, \ldots \\ 4, n=7,9,11, \ldots\end{array}\right.$

\section{Proof:}

Let $G=W_{n}$ be a wheel graph.

Let $u_{1}$ be the root vertex of $G$.

Let $v_{1}, v_{2}, \ldots . v_{n}$ be the main vertex of $G$.

2- Dominator coloring of $G$ as follows.

Case 1: $\chi_{d, 2}\left(W_{n}\right)=3, n=6,8,10, \ldots$

First color $c_{0}$ to the root vertex $u_{1}$. Assign color $, C_{1}, C_{2}{ }^{\text {ee }}$ alternatively to the remaining verticies of $G$. i.e $v_{1} v_{2}$ receive color $C_{1} C_{2}, v_{2} v_{3}$ receive color $C_{2} C_{1}, v_{3} v_{4}$ will receive $C_{1} C$ and so on. By coloring it forms a clique. Hence every vertex must dominate at least two color classes. Thus $\chi_{d, 2}\left(W_{n}\right)=$ $3, n=6,8,10, \ldots$

Case 2: $\chi_{d, 2}\left(W_{n}\right)=4, n=7,9,11, \ldots$

Assign color $C_{0}$ to the root vertex $u_{1}$. Now the colors $C_{1} C_{2}, C_{3}$ will be assigned alternatively to the remaining vertices of $\mathrm{G}$. i.e. $v_{1} v_{2}$ by $C_{1} C_{2}, v_{2} v_{3}$ by $C_{2} C_{3}, v_{3} v_{4}$ by $C_{3} \mathrm{C}_{1}, v_{4} v_{5}$ by $C_{1} C_{2, \ldots \ldots, \text { and }}$ so on.By coloring it forms a clique while assigning the color in the above alternative sequence, at last there may or may not remains some vertices of $G$, it depends up on ,n n $^{\text {e }}$ suppose the vertices of $G$ remains, to color the remaining vertices of $G$ using the above same three colors $c_{1} c_{2}, c_{3}$ colored in any order, but there should not any two adjacent vertices receive the same color. Hence every vertex must dominate at least two color classes. Thus $\chi_{d, 2}\left(W_{n}\right)=4, n=7,9,11, \ldots$

\section{Theorem 2.10:}

For any prism graph $Y_{2, n}, \chi_{d, 2}\left(Y_{2, n}\right) \leq n, \mathrm{n}=3,4,5, \ldots \ldots$

Proof:

Let $G=Y_{2, n}$ be a prism graph. Let $G=U \cup V$. Let $U=$ $\left\{u_{0}, u_{1}, u_{2}, \ldots ., u_{n-1}\right\}$ be the inner vertices of $G . V=\left\{v_{0}\right.$ $\left.v_{1}, v_{2}, \ldots . . v_{n-1}\right\}$ be the outer vertices of $G$ respectively. Let $E(U)=\left\{u_{i} u_{i+1} ; 0 \leq i \leq n-2\right\}$ and $E(V)=\left\{v_{i} v_{i+1} ; 0 \leq i \leq\right.$ $n-2\}$. Thus for any $Y_{2, n}, E(G)=3 n$. Here $x=1$ be the edge joining the vertex of $U$ and $V$.

Let color the vertices of $U$ be $C=\{0,2 x+1,2(2 x+1), \ldots,(M-$ $1)(2 \mathrm{I}+1)\}$ by $M$ different colors. Note $M=\lceil\mathrm{n} /(2 \mathrm{x}+1)\rceil$, $x=1$ be the edge joining the vertices of $U$ colored by another different colors $1,2, \ldots, \mathrm{n}-\mathrm{M}$ in clockwise direction respectively. Similarly the vertices of $V$ can be colored by the above said same $M$ colors, but it starts from the vertices $v_{1}$, it may not be end at the vertex $v_{0}$.i.e., $v_{1}, v_{4}, v_{7}, \ldots$ And so on. Also the remaining vertices of $V$ colored by above said same coloring using same concept. Therefore by coloring, there exists a clique between the vertices $U$ and $V$ of $G$. Thus, $G$ dominates at least two color classes. Hence $G$ admits $\chi_{d, 2}\left(Y_{2, n}\right) \leq n, \mathrm{n}=3,4,5, \ldots \ldots$ 


\section{International Journal of Science and Research (IJSR)}

ISSN (Online): 2319-7064

Index Copernicus Value (2013): 6.14 | Impact Factor (2015): 6.391

Theorem 2.11

For any graph $G, G=W_{3, n}$ then $\chi_{d, 2}(G) \leq n, n \geq 3$.

\section{Proof:}

Let $W_{n}$ be a web graph.

Let $G=U \cup V \cup W$ be the vertex set.

Let $U=\left\{u_{0}, u_{1}, \ldots \ldots, u_{n-1}\right\} \quad V=\left\{v_{0}, v_{1}, \ldots \ldots, v_{n-1}\right\}$ and $W=\left\{w_{0}, w_{1}, \ldots \ldots, w_{n-1}\right\}$ respectively.

Let $E(U)=\left\{u_{i} u_{i+1} ; 0 \leq i \leq n-2\right\}$ and $E(V)=\left\{v_{j} v_{j+1}\right.$; $0 \leq j \leq n-2\}$ be the edge set of $U$ and $V$

Let $\mathrm{E}(\mathrm{t})=\left\{\begin{array}{l}u_{k} v_{k} ; 0 \leq K \leq n-1 \\ v_{k} w_{k} ; 0 \leq K \leq n-1\end{array}\right.$ be the edge between the vertex set $U V$ and $V W$ respectively. Thus for any web graph $W_{3, n} E(G)=3 n+n$. Consider $x=1$ be the edge joining the vertex $U V$ and $V W$.

Now the procedure for 2 -dominator coloring of $\mathrm{G}$ follows:

Let color the vertex $G \in U \cup V \cup W$ by $C=\{0,2 x+1,2(2 x+1), \ldots .,(M-1)(2 x+1)\}$ by $M$ different colors

in clockwise direction. Note $M=\lceil\mathrm{n} /(2 \mathrm{x}+1)\rceil, x=1$. Also the remaining vertices of $G \in U \cup V \cup W$ colored by $\mathrm{n}-M$ different color $1,2, \ldots, n-M$ in clockwise direction. To color the vertices of $U$, starts from the vertex $u_{0}$ using $C=\{$ $0,2 x+1,2(2 x+1)\}, \ldots .(M-1)(2 \mathrm{x}+1)\}$ and it may or may not ends at the vertex $u_{n-1}$ and the remaining verticies of $\mathrm{G}$ colored by $n-M$ different colors. Next color the vertices of $V$ starts from vertex $v_{1}$ using above said the $M$ colors $C=\{$ $0,2 x+1,2(2 x+1)\}, \ldots \ldots . .(M-1)(2 x+1)\}$ and it also may or may not ends at the vertex $v_{n-1}$ then the remaining vertices of $\mathrm{v}$ colored by the same said $n-M$ different colors in same previous manner. Similarly to color the remaining vertices of $W$ needs those same $n-M$ colors using same procedure as said above. Likewise coloring there exist a cliques in $G$.

Hence every vertex of $\mathrm{G}$ dominates at least two color classes. Thus for any $W_{n}, \chi_{d, 2}\left(W_{3, n}\right) \leq M+n-m \leq n, n \geq 3$.

\section{Theorem 2.12}

If $G=H_{n}$ ba a helm graph, then

Proof:

$$
\chi_{d, 2}\left(H_{n}\right)=\left\{\begin{array}{c}
3, n=6,8,10,12, \ldots \\
4, n=7,9,11, \ldots .
\end{array}\right.
$$

By the above theorem $\chi_{d, 2}\left(W_{n}\right)=\left\{\begin{array}{c}3, n=6,8,10,12, \ldots \\ 4, n=7,9,11, \ldots .\end{array}\right.$ definition of helm graph, assign color $c_{0}$ to all the pendent vertices $w_{0}, w_{1}, \ldots \ldots, w_{n}$ in $\mathrm{G}$, which was already colored to the root vertex $u_{0}$ in $\mathrm{G}$. By definition of 2-dominator coloring $\chi_{d, 2}\left(H_{n}\right)=\left\{\begin{array}{c}3, n=6,8,10,12, \ldots \\ 4, n=7,9,11, \ldots \ldots\end{array}\right.$

\section{Cayley Graph}

Let $G$ be a group, and let $S$ be subset of $G$. Then the Cayley Digraph $D(G, S)$ on $G$ with connection set $S$ is defined as follows:

1. The vertices are the elements of $G$

2. There is an arc joining $g$ and $h$ if and only if $h=s_{g}$ for some $s \in S$.

We can extend this idea to a Cayley Color graph, where $S$ is a generating set for $G$, each $s_{i} \in \mathrm{S}$ is assigned a color, and if $g$

$=s_{i h}$, then the arc connecting them is colored $s_{i}$.

\section{Theorem 2.12}

Let $G$ be a group with generating set $S$ and let $\varphi$ be a colorpreserving permutation on $V(D(G, S))$. Then $\varphi$ is a color preserving automorphism of $D(G, S)$ if and only if $\varphi(g h)=$ $\varphi(g) h$.

Suppose that $\varphi(g h)=\varphi(g) h$.

Proof:

To show that $\varphi$ is color-preserving, we need to show that if $g h-1=s$, then $\varphi(g h-1)=s$. Suppose $g h-1=s$. Then $\varphi(g h-1$ )$=\varphi(g) h-1=\varphi(g) g-1 s=\varphi(g g-1) s=s$.

\section{Conclusion}

In this paper, we found out some result of various graphs like,Central Graph of Star Graph C [ $\left.S_{1, n}\right]$, Star Graph: $S_{1, n}$, Barbell Graph B $\left(K_{n}, K_{n}\right)$, Ladder Graph, Banana Tree $B_{m, n}$, Fire cracks graph: $F_{n, k}, \mathrm{n}, \mathrm{k} \geq 2$, Wind Mill Graph $D_{n}^{(m)}$, Gear Graph $G_{n}$, Wheel Graph, Prism Graph $Y_{2, n}$, Web Graph $W_{n}$, Helm Graph $H_{n}$, Cayley Graph using 2-dominator coloring.This will be initiative study of extension of 2 Dominator coloring.

\section{References}

[1] S. Arumugam, Jay Bagga and K. RajaChandrasekar, 2012,"On Dominator ColoringsinGraphs", Proc. Indian Acad. Sci. (Math. Sci.)Vol. 122, No. 4, November, pp. 561-571.

[2] E.Cockayne,S.HedetniemiandS.

Hedetniemi, "Dominating partitions of Graphs", unpublished manuscript.

[3] R. Gera, S Horton, C. Rasmussen, "Dominator Colorings and Safe Clique Partitions", Congressus Numerantium, 2006.

[4] S. M. Hedetniemi, S. T. Hedetniemi, and A. A. McRae, Dominator Colorings of graphs, (2006). In preparation.

[5] T. W. Haynes, S. T. Hedetniemi, and P. J. Slater, "Domination in Graphs: Advanced Topics", Marcel Dekker, New York, 1998.

[6] Marcel Dekker, "Fundamentals of Domination in Graphs", New York, 1998.

[7] Ralucca Michelle Gera, On Dominator Colorings in Graphs, Department Of Applied Mathematics, Naval Postgraduate School, Monterey, CA 93943, USA.

[8] T.Ramachandran, A.Naseer Ahmed, "Dominator Coloring Number of Middle Graph", IOSR Journal of Mathematics, Vol. 10, Iss. 6, Ver. IV, pp 38-40, December 2014.

[9] A.Sangeetha Devi,M.M.Shanmugapriya, 2-Dominator Coloring in Graphs,International Journal of Applied Engineering Research,Vol. 10, 43059-43062.2015,

[10]E. Sampathkumar and L. Pushpa Latha, "Strong, weak Domination and Domination Balance in a Graph", Discrete Math. 161.235-242 (1996). 